\title{
Current Disposable Ureteroscopes: Performance and Limitations in a Standardized Kidney Model
}

\author{
Daniel Schlager, MD, Moaaz Abdulghani Obaid, MD, Simon Hein, MD, Konrad Wilhelm, MD, \\ Martin Schönthaler, MD, PhD, Christian Gratzke, MD, PhD, \\ Arkadiusz Miernik, MD, PhD, and Dominik Stefan Schoeb, MD
}

\begin{abstract}
Objectives: Due to the increasing fragility of the instruments and rising concerns about the sterility of reprocessable scopes, several single-use devices for flexible ureteroscopy have been introduced. In this study, we compare currently available disposable digital and fiberoptic flexible ureteroscopes with a contemporary reusable fiberoptic device.

Materials and Methods: LithoVue ${ }^{\mathrm{TM}}$, Pusen Uscope ${ }^{\circledR}$ (UE3011, UE3022), Flexor ${ }^{\circledR}$ Vue $^{\mathrm{TM}}$, and a reusable fiber optic flexible ureteroscope (BOA vision ${ }^{\circledR}$ ) were tested in kidney models. The setup included (1) visualization of all calices (correct assignment of colored pearls) and (2) the extraction of human calculi with a standard disposable extraction device $\left(\mathrm{NGage}^{\circledR}\right)$. We documented the effective visualization, stone extraction, and times to completion. In addition, the surgeons' workload and performance were determined using the National Aeronautics and Space Administration-Task Load Index.

Results: In visualizing and identifying calices, the LithoVue and both generations of the Uscope performed similarly, but time to completion was significantly longer for all single-use devices in comparison with the Boa Vision. LithoVue retracted stones almost as well as the reusable scope (97\% vs 95\%/82\%/96\% stone clearance), while accessibility was impeded using Uscope UE3011, as reflected by the retrieval time per stone (73 vs 102 seconds/stone). This disadvantage has, however, been overcome with the new Uscope Generation UE3022, showing a retrieval time of only 65 seconds per stone, excelling over the reusable scope in this category. The Uscope UE3022 image quality was also rated best, but showed no significant difference.

Conclusions: In comparison with disposable ureteroscopes available, LithoVue offers performance and characteristics similar to a reusable device, while the FlexorVu's performance does not yet yield satisfactory results for clinical use. The first generation of Uscope exhibits potential, but requires further technical improvements to match the performance of a reusable device. With the new-generation UE3022, Pusen has made significant improvement and offers a quality comparable with the LithoVue's.
\end{abstract}

Keywords: disposable equipment, fiber optic technology/instrumentation, humans, kidney calculi/diagnostic imaging*, kidney calculi/surgery, ureteroscopes*, ureteroscopy/instrumentation*

\section{Introduction}

D URING THE PAST DECADES, TREATMENT of upper urinary tract pathologies has been revolutionized by technologic progress. Especially in the management of upper urinary tract stone disease, a paradigm shift has occurred. Extracorporeal shock wave lithotripsy has largely been replaced by an endoscopic approach, ${ }^{1}$ much due to the tremendous im- provement in image quality and maneuverability of the ureteroscopes currently available.

In recent years, the field of flexible ureteroscopy (fURS) has witnessed a trend toward the application of disposable devices to reduce the inconsistent performance and high maintenance costs due to repairs and necessary sterilization. ${ }^{2-4}$ While these devices do not constitute an immediate improvement in performance and quality, they can improve

Department of Urology, Medical Center-University of Freiburg, Faculty of Medicine, University of Freiburg, Germany.

Meetings at which the work has been presented, World Congress of Endourology 2018 (in part), Vancouver, Canada. 
workflow (no time-intensive sterilization, no repair costs) and potentially minimize infection rates by reducing devicerelated bacteria transfer. ${ }^{5}$ In our previous study, we evaluated one of the first commercially available semidisposable endoscopic systems, the Flexor ${ }^{\circledR}$ Vue ${ }^{\mathrm{TM}}$ (Cook Medical, Bloomington, IN). ${ }^{6}$ Our data revealed that technical improvements are still necessary for the FlexorVue system to qualify as a replacement for currently available reusable scopes.

Since then, new disposable ureterorenoscopes have become available, We now present our updated data evaluating all commercially available single-use devices, namely the LithoVue $^{\mathrm{TM}}$ system (Boston Scientific, Marlborough, MA) as well as the Pusen Uscope ${ }^{\circledR}$ system UE3011 and UE3022 (Clarion Medical Technologies, Inc., Cambridge, Canada) in an artificial kidney model using a standardized experimental setup.

\section{Materials and Methods}

\section{Tested endoscopic devices}

In this study, four commercially available single-use endoscopes for fURS were evaluated: the LithoVue system by Boston Scientific, the Pusen Uscope UE3022 system by Clarion Medical Technologies, Inc. and its predecessor the Pusen Uscope UE3011, which has the same dimensions and deflection as the current version, but is designed with a semiflexible shaft and a flexible tip only. Also, the design of the handle differs; the UE3011 offers a pistol-like handle, while the UE3022 is ergonomically similar to standard endoscopic designs. As a reference device, we used a standard reusable fiber optic flexible ureteroscope (BOA vision ${ }^{\circledR}$; Richard Wolf GmbH, Knittlingen, Germany). The technical characteristics of all used endoscopes are depicted in Table 1.

\section{Experimental setting and trial setup}

As described previously, ${ }^{6}$ our experimental setup included two basic tasks: standardized visualization of colored pearls (resembling kidney stones) to assess handling and the freedom of movement to maneuver within the kidney. Second, the extraction of stones (2-3 mm length) with a standard stone-extracting device (NGage ${ }^{\circledR}$; Cook Medical). These tasks were performed by the same six endourology-trained surgeons with different levels of experience in endourology (two attending, two fellows, and two residents). Although the experience level between participants did vary according to their time in practice, all surgeons had performed a high number of clinical interventions before the study and were familiar with applying conventional ureteroscopes for stone removal. The kidney models were fixed to the bottom of a $33 \times 68 \times 11 \mathrm{~cm}$ plastic basin that was filled with saline. An opening was created in the basin wall toward the surgeon and coated with a sealing ring. The kidney models were connected via ureteral access sheaths (Flexor Ureteral Access Sheath, 12F, $35 \mathrm{~cm}$; Cook Medical) as used in procedures with patients. This experimental setup is depicted in Figure 1. All tasks were performed with each ureteroscope once for every kidney model. A new reusable endoscope device was used for each task and for every surgeon. The surgeons had 15 minutes to complete each task. To avoid any training effect or influence on the surgeons' perception, procedures were carried out on different days. To rule out their ability to compare the first instrument or the image seen, the order in which each surgeon was given an ureteroscope was randomly assigned using a simple numbers lottery.

Since all devices possess distinct features and are easily distinguishable, it was not possible to blind the participants. Once the instruments had been randomly assigned, each surgeon was given time to familiarize himself with the device until considered fit for trial. For the first task, surgeons were asked to navigate through the kidney model and identify the calices by number. A picture of the kidney model with the numbered calices was provided to the surgeon throughout the task. To facilitate correct internal verification, each calix was marked with a different-colored pearl combination.

\section{Data collection}

We documented the effective visualization of calices, stone extraction, and times to completion. In addition, the surgeons' workload and performance were determined using the National Aeronautics and Space Administration (NASA)Task Load Index (TLX; Supplementary Fig. S1), a standardized, subjective, multidimensional assessment tool for perceived workload developed by the NASA's Human performance group. It evaluates the perceived workload in the following categories: mental demand, physical demand,

Table 1. Technical Characteristics of All Endoscopes Used in This Trial

\begin{tabular}{|c|c|c|c|c|c|}
\hline Characteristics & Boa Vision & Flexor $^{\circledR}$ Vue $^{\mathrm{TM}}$ & LithoVue & $\begin{array}{c}\text { Pusen Uscope }{ }^{\circledR} \\
\text { (UE3011) }\end{array}$ & $\begin{array}{l}\text { Pusen Uscope } \\
\text { (UE3022) }\end{array}$ \\
\hline Manufacturer & Richard Wolf & Cook medical & Boston Scientific & Clarion Medical & Clarion Medical \\
\hline Type of use & Reusable & Single use & Single use & Single use & Single use \\
\hline Optical system & Fiber optic & $\begin{array}{l}\text { Fiber optic } \\
\quad \text { (reusable) }\end{array}$ & $\begin{array}{l}\text { Digital CMOS } \\
\text { imager }\end{array}$ & $\begin{array}{l}\text { Digital CMOS } \\
\text { imager }\end{array}$ & $\begin{array}{l}\text { Digital CMOS } \\
\text { imager }\end{array}$ \\
\hline Length (mm) & 680 & 450 & 680 & 650 & 650 \\
\hline Working channels & 1 & $2^{\mathrm{a}}$ & 1 & 1 & 1 \\
\hline Working channel diameter & $3.6 \mathrm{~F}$ & $3 \mathrm{~F} / 9 \mathrm{~F}$ & $3.6 \mathrm{~F}$ & $3.6 \mathrm{~F}$ & $3.6 \mathrm{~F}$ \\
\hline Tip diameter & $6.6 \mathrm{~F}$ & n.a. & $7.7 \mathrm{~F}$ & $9.0 \mathrm{~F}$ & $9.0 \mathrm{~F}$ \\
\hline Shaft diameter & $8.7 \mathrm{~F}$ & $15 \mathrm{~F}^{\mathrm{b}}$ & $9.5 \mathrm{~F}$ & $9.5 \mathrm{~F}$ & $9.5 \mathrm{~F}$ \\
\hline Deflection & $\begin{array}{l}\text { Bidirectional } \\
270^{\circ} / 270^{\circ}\end{array}$ & $\begin{array}{l}\text { Unidirectional } \\
180^{\circ} \%\end{array}$ & $\begin{array}{l}\text { Bidirectional } \\
270^{\circ} / 270^{\circ}\end{array}$ & $\begin{array}{l}\text { Bidirectional } \\
270^{\circ} / 270^{\circ}\end{array}$ & $\begin{array}{l}\text { Bidirectional } \\
270^{\circ} / 270^{\circ}\end{array}$ \\
\hline
\end{tabular}

\footnotetext{
${ }^{\mathrm{a}}$ One working channel is required for optical fiber.

${ }^{\mathrm{b}}$ Shaft includes access sheath.

CMOS $=$ complementary metal-oxide-semiconductor; $\mathrm{n} . \mathrm{a} .=$ not available .
} 


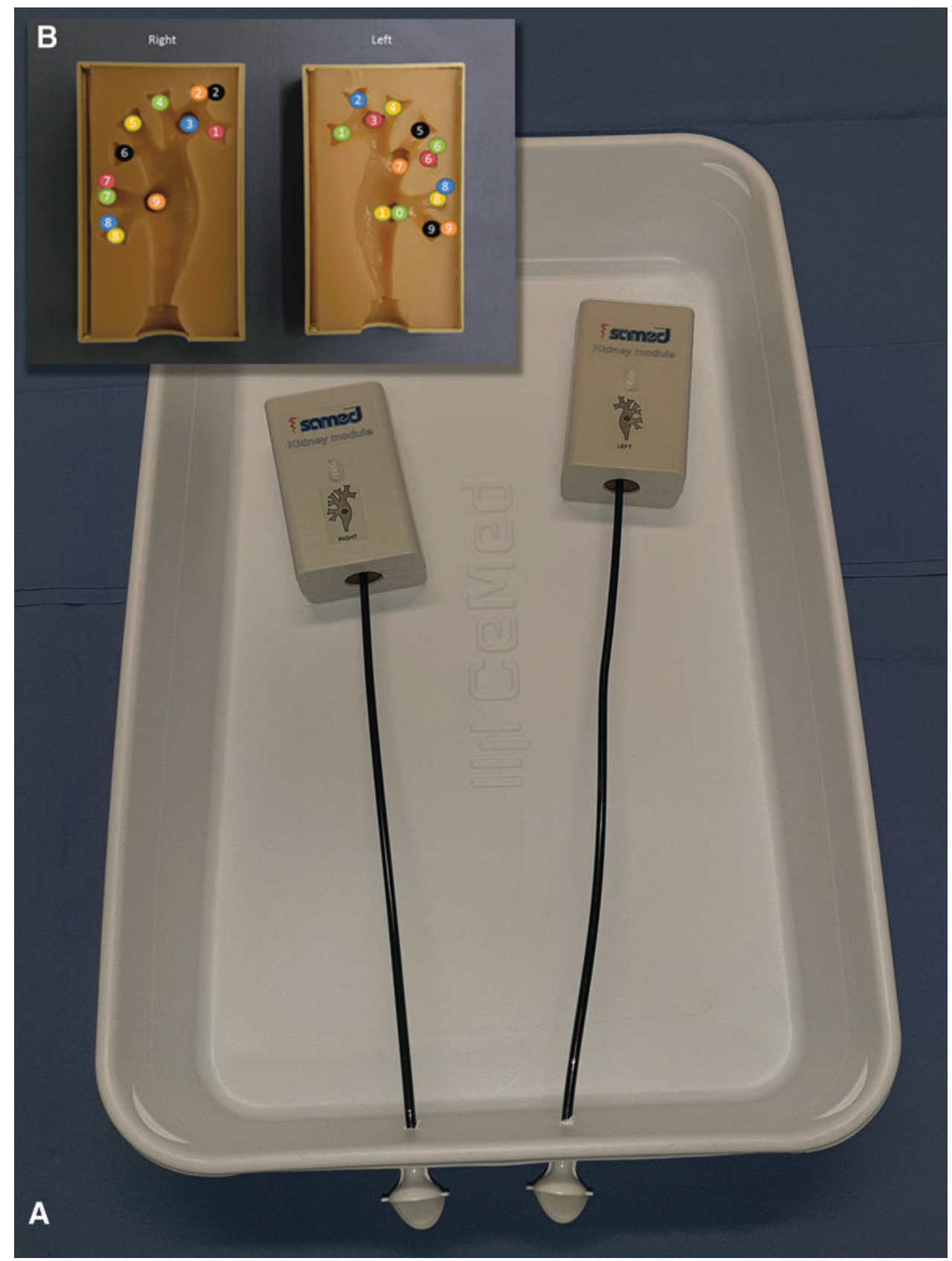

FIG. 1. Experimental setup used in this study. (A) Kidney models, fixed to the bottom of a $33 \times 68 \times 11 \mathrm{~cm}$ plastic basin. (B) Artificial calix system of the kidney model with pearls. Numbers indicate calices; colored background indicates color of pearl placed in calix.

temporal demand, performance, effort, and frustration. In addition, we referred to a Likert scale to assess maneuverability, handling, and image quality. Here, surgeons were asked to rank the respective feature according to their experience with the device from 1 ("very poor") to 5 ("very good").

\section{Statistical evaluation}

Endpoints were evaluated using Wilcoxon's rank-sum test (baseline comparison of weight of probes and comparison of all nonparametric endpoints) and Fishers' exact test (binary parameters). Sample size calculation was based on our previous study. ${ }^{6}$ All statistical computations were performed using Python 2.7 (Enthought, Austin, TX; Canopy distribution 1.1.0.1371; scipy statistical package). The test used in each case is indicated by superscript letters. $p$-Values $<0.05$ were considered to be significant.

\section{Results}

\section{General characteristics}

Dimensions and specifications of the applied ureteroscopes in this study are listed in Table 1. All devices offered one working channel for the application of grasping devices or laser fibers, with the exception of the FlexorVue (3F) working channel diameter being 3.6F. The FlexorVue can be described as a semidisposable device with a disposable deflecting ureteral sheath and inner optics that can be reused as much as nine times. It provides an outer diameter of $15 \mathrm{~F}$, but that already includes the built-in access sheath. Working 
length of both generations of the Uscope was $650 \mathrm{~mm}$, while the Boa Vision and FlexorVue were a little longer with $680 \mathrm{~mm}$. The FlexorVue device is, however, significantly shorter, as its working length measures only $450 \mathrm{~mm}$. Also, the FlexorVue device is the only unidirectional device with a $180^{\circ}$ flection in one direction, while all other devices offer bidirectional $270^{\circ}$ flection. The Uscopes enable the surgeon to choose standard or reverse deflection; reverse deflection was utilized in our study. The LithoVue and both generations of Uscope offer a digital optical system [complementary metaloxide-semiconductor (CMOS) image] with a built-in light source, while the reusable Boa Vision device and FlexorVue device use a fiber optic in this study. The LithoVue system comprises a workstation, including a monitor mounted on a mobile cart with integrated image-processing software, but it is also possible to connect the device to a digital visual interface (DVI) monitor in the operation theater. The camera head is integrated in the plastic handle and comes with a CMOS imager. The Pusen Uscope can be operated via an additional touch-screen monitor or via the operating room's built-in DVI monitors. The camera head is integrated in the head as well. Noticeably, both uroscopes' $9.0 \mathrm{~F}$ tip size is the largest in this study. All tested devices are depicted in Figure 2.

\section{Visualization and image quality}

Results of visualization and image quality from all trials all summarized in Table 2. Visualization of all calices was achieved in $100 \%$ and correct identification of calices was achieved in $99 \%$ of cases with our reusable reference device (Boa Vision). Average time until completion was $153 \mathrm{sec}-$ onds. All reusable devices showed an acceptable visualization/identification rate with 99/97\% for LithoVue, 100/100\% for the Pusen Uscope (UE3011), and 97/89\% for the Pusen Uscope (UE3022) except for the FlexorVue with only 74/ $81 \%$. However, time to completion was significantly longer for all devices in comparison with the reference scope. Ranking of image quality between the devices showed no significant difference, except for a significantly lower quality for the FlexorVue $(p<0.001)$. Equally, handling was ranked without a significant difference except for the FlexorVue $(p<0.001)$. However, a slight advantage for the LithoVue and the Pusen Uscope (UE3022) regarding handling and maneuvering in the kidney was observed with an average grade on the Likert scale of 1.83 for both devices compared with 3.17 for the Pusen Uscope (UE3011). The Boa Vision's handling was ranked highest with an average grade of 1.33. Maneuverability of all the single-use instruments was ranked significantly lower than the reference devices', except for the Pusen Uscope (UE3022). For the latter, the average grade of 1.92 (compared with the Boa Vision's 1.58) was still lower but without a significant difference.

The NASA-TLX analysis rated the Boa Vision highest, giving it an overall average of 3.94 compared with 6.37 (LithoVue), 13.8 (FlexorVue), 9.79 (Pusen Uscope, UE3011), and 5.01 (Pusen Uscope, UE3022). However, we noted a significantly lower ranking in only one category (physical demand), for the LithoVue, and one category (frustration), for the Pusen Uscope UE3022, revealing almost equivalent ratings in the NASA-TLX for these devices.

\section{Stone extraction}

Similar to the results for visualization/identification as reported above, stone clearance rates were similar for the Boa Vision (97\%), LithoVue (95\%), and the Uscopes (UE3011 $82 \%$ and UE3022 96\%) as summarized in Table 3. The FlexorVue device, however, only showed a clearance rate of $23 \%$, and its clearance time was significantly slower than the Boa Vision's (331 seconds, $p=0.04$ ). Interestingly, while not significantly different, the Uscope UE3022 showed the fastest retrieval time per stone with 65 seconds (s) compared with 73 seconds for the Boa Vision, 88 seconds for the LithoVue, and 102 seconds for the Uscope UE3011.

We documented no significant differences in the maneuverability and handling of the single-use devices when extracting stones, except that the FlexorVue performed significantly worse $(p<0.001)$. However, the Boa Vision yielded the best average grade with 1.83/1.83 compared with 2.07/2.43 (LithoVue), 3.33/3.50 (Uscope UE3011), and 1.91/ 2.08 (Uscope UE3022) on the Likert visual analog scale. NASA-TLX evaluation showed an overall average of 5.81 compared with 8.65 (LithoVue), 17.31 (FlexorVue), 11.10 (Pusen Uscope, UE3011), and 7.25 (Pusen Uscope, UE3022). In this context, the Uscope UE3022 was ranked significantly lower than the Boa Vision only for mental demand; the UE3022 was similar in all other categories.

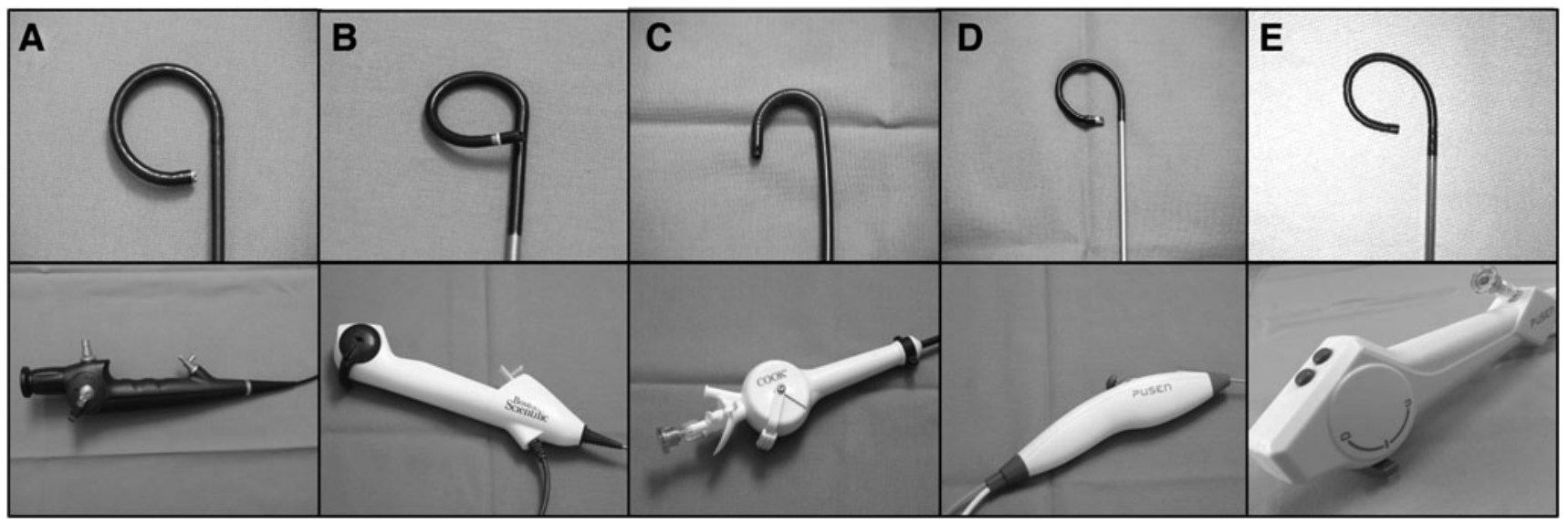

FIG. 2. Endoscopes in full deflection and according handpieces below: (A) Boa Vision, (B) LithoVue, (C) FlexorVue, (D) Uscope UE3011, and (E) Uscope UE3022. 
Table 2. Results of Visualization and Workload Assessment for All Trials

\begin{tabular}{|c|c|c|c|c|c|}
\hline & Boa Vision & LithoVue & FlexorVue & $\begin{array}{l}\text { Pusen Uscope } \\
\text { (UE3011) }\end{array}$ & $\begin{array}{l}\text { Pusen Uscope } \\
\text { (UE3022) }\end{array}$ \\
\hline \multicolumn{6}{|l|}{ Parameters } \\
\hline Calices visualized & $100 \%(114 / 114)$ & $99 \%(113 / 114)$ & $74 \%(84 / 114)$ & $100 \%(114 / 114)$ & $97 \%(111 / 114)$ \\
\hline Calices correctly identified & $99 \%(113 / 114)$ & $97 \%(111 / 114)$ & $81 \%(68 / 84)$ & $100 \%(114 / 114)$ & $89 \%(102 / 114)$ \\
\hline Time (seconds) to completion & 153 & $275(\mathbf{0 . 0 4 8})$ & $755(<\mathbf{0 . 0 0 1})$ & $319(\mathbf{0 . 0 0 3})$ & $416(\mathbf{0 . 0 0 3 )}$ \\
\hline Image quality (1-5, mean) & 1.83 & $1.75(0.2)$ & $2.92(<\mathbf{0 . 0 0 1})$ & $2.67(0.48)$ & $1.58(0.207)$ \\
\hline Handling $(1-5$, mean $)$ & 1.33 & $1.83(0.073)$ & $4.00(<\mathbf{0 . 0 0 1})$ & $3.17(0.40)$ & $1.83(0.82)$ \\
\hline Maneuverability ( $1-5$, mean) & 1.58 & $2.21(\mathbf{0 . 0 0 2})$ & $4.08(<\mathbf{0 . 0 0 1})$ & $3.17(<\mathbf{0 . 0 0 1})$ & $1.92(0.409)$ \\
\hline \multicolumn{6}{|l|}{ NASA-TLX } \\
\hline Mental demand (1-20, mean) & 4.33 & $6.83(0.37)$ & $15.17(<\mathbf{0 . 0 0 1})$ & $11.83(\mathbf{0 . 0 0 2})$ & $6.16(0.209)$ \\
\hline Temporal demand $(1-20$, mean $)$ & 4 & $6.58(0.65)$ & $12.33(<0.001)$ & $10.58(\mathbf{0 . 0 2 2})$ & $4.08(0.948)$ \\
\hline Performance $(1-20$, mean) & 3.17 & $6.50(0.79)$ & $12.42(<\mathbf{0 . 0 0 1})$ & $9.92(0.089)$ & $4.75(0.275)$ \\
\hline Effort $(1-20$, mean $)$ & 5.58 & $4.33(0.37)$ & $16.58(<\mathbf{0 . 0 0 1})$ & $5.67(\mathbf{0 . 0 2})$ & $7.25(0.368)$ \\
\hline Frustration (1-20, mean) & 2.50 & $8.75(0.58)$ & $15.25(<\mathbf{0 . 0 0 1})$ & $11.08(<\mathbf{0 . 0 0 1})$ & $5.33(<\mathbf{0 . 0 0 1})$ \\
\hline Physical demand (1-20, mean) & 4.08 & $6.58(\mathbf{0 . 0 4 3})$ & $10.92(<\mathbf{0 . 0 0 1})$ & $9.67(\mathbf{0 . 0 1 4})$ & $3.08(0.133)$ \\
\hline TLX overall (mean) & 3.94 & 6.37 & 13.8 & 9.79 & 5.01 \\
\hline
\end{tabular}

The $p$-values for the comparison with the Boa Vision device are given in brackets for each value. Significant differences are in bold. NASA $=$ National Aeronautics and Space Administration; TLX = Task Load Index.

\section{Discussion}

The present study provides an update to our previously published data on the in vitro evaluation of single-use flexible ureteroscopes compared with a standard reusable scope. We tested all commercially available single-use ureteroscopes at the time of the study. Our data reveal similar performance levels for the LithoVue and Uscope UE3022 when compared with a reusable scope. While the LithoVue revealed a slight advantage in the visualization and identification of calices and in handling during this step, the Uscope UE3022's performance was superior during the stone removal process, with its even faster removal time per stone than the reference device. The Uscope UE3011 was found to be inferior to its successor mainly in usability terms (NASA-TLX). This problem has been solved in the new-generation UE3022 mainly due to the change from a pistol-like handle to the classic design of a standard ureteroscope handle. We believe that the main reason for its superior performance during stone removal might be the surgeon's familiarity with the classically designed ureteroscope handle. Accordingly, the UE3022's removal time compared with the LithoVue, which also uses a handle of "classic" design, only differed slightly, implying an advantage for the standard handle. As reported previously, 6 the FlexorVue's performance is inadequate for routine clinical use as an addition to or even replacement for current reusable ureteroscopes.

Altogether, the performances of all the devices we tested were still inferior to that of a state-of-the-art reusable device, but the new LithoVue and Uscope, especially its newest edition UE3022, deliver remarkable image quality and good intraprocedural performance, which is congruent with other trial results on this subject. ${ }^{7}$ They offer a genuine, costeffective alternative to having a reusable device at hand at all times especially for clinical centers that have low-volume intrarenal surgery cases. Furthermore, since reusable devices are susceptible to damage especially whenever manipulation at maximum flection is necessary or a large stone mass must be treated, single-use devices might pose an alternative as well. Our study, however, has some limitations. First, the

Table 3. Results of Performance and Workload Assessment for All Trials

\begin{tabular}{lccccc}
\hline & Boa Vision & LithoVue & FlexorVue & Uscope UE3011 & Uscope UE3022 \\
\hline Parameters & & & & & \\
$\quad$ Calices cleared & $97 \%(111 / 114)$ & $95 \%(108 / 114)$ & $23 \% 26 / 114$ & $82 \%(94 / 114)$ & $96 \% 109 / 114$ \\
Retrieval time per stone (seconds) & 73 & $88(0.378)$ & $331(\mathbf{0 . 0 4})$ & $102(0.13)$ & $65(0.506)$ \\
Handling (1-5, mean) & 1.83 & $2.07(0.3)$ & $4.58(<\mathbf{0 . 0 0 1})$ & $3.33(0.1)$ & $1.91(0.813)$ \\
Maneuverability (1-5, mean) & 1.83 & $2.43(0.12)$ & $4.58(<\mathbf{0 . 0 0 1})$ & $3.50(0.13)$ & $2.08(0.277)$ \\
NASA-TLX & & & & & \\
Mental demand (1-20, mean) & 6 & $9.36(\mathbf{0 . 0 1 3})$ & $16.67(<\mathbf{0 . 0 0 1})$ & $11.67(0.74)$ & $8.33(\mathbf{0 . 0 6 2})$ \\
Physical demand (1-20, mean) & 6.08 & $9.36(\mathbf{0 . 0 0 4})$ & $15.50(<\mathbf{0 . 0 0 1})$ & $11.17(0.055)$ & $6.08(0.431)$ \\
Temporal demand (1-20, mean) & 6.67 & $8.64(\mathbf{0 . 0 0})$ & $16.83(<\mathbf{0 . 0 0 1})$ & $11.50(\mathbf{0 . 0 3 5})$ & $6.50(0.41)$ \\
Performance (1-20, mean) & 4.67 & $6.50(0.20)$ & $17.50(<\mathbf{0 . 0 0 1})$ & $8.92(\mathbf{0 . 0 4 6})$ & $6.00(0.766)$ \\
Effort (1-20, mean) & 6.67 & $10(0.05)$ & $18.50(<\mathbf{0 . 0 0 1})$ & $11.50(\mathbf{0 . 0 2})$ & $8.75(0.560)$ \\
Frustration (1-20, mean) & 4.75 & $8.07(\mathbf{0 . 0 4})$ & $18.83(<\mathbf{0 . 0 0 1})$ & $11.83(\mathbf{0 . 0 0 3})$ & $7.83(0.194)$ \\
TLX overall (mean) & $\mathbf{5 . 8 1}$ & $\mathbf{8 . 6 5}$ & $\mathbf{1 7 . 3 1}$ & $\mathbf{1 1 . 1 0}$ & $\mathbf{7 . 2 5}$ \\
\hline
\end{tabular}

The $p$-values for the comparison with the Boa Vision device are given in brackets for each value. Significant differences are in bold. 
in vitro setting cannot simulate the impact of contamination in the irrigation fluid due to blood or stone fragments on the image quality. We also did not simulate further clinical characteristics such as the loss of irrigation fluid or maneuverability with a laser fiber. The goal of our study was to assess the handling of available devices in an experimental setting to assess general applicability for clinical use. The actual performance of those new devices that showed potential is being further evaluated in a clinical trial. Furthermore, our study was performed in a high-volume center by surgeons accustomed to using reusable ureteroscopes, which could cause a potential bias and enable a better evaluation of the reference device. In addition, after we concluded our experiments, another single-use flexible ureteroscope, the Dornier Axis $^{\mathrm{TM}}$ (Dornier MedTech Europe GmbH, Weßling, Germany), was launched, which we were thus unable to investigate in this study.

\section{Conclusions}

In this study, we report on our in vitro evaluation of currently available single-use flexible ureteroscopes. In comparison with the disposable ureteroscopes currently available, the LithoVue offers overall a performance and characteristics similar to a standard reusable device, while the FlexorVue's performance failed to deliver satisfactory results for routine clinical use. The first generation of Uscope exhibits potential for upper urinary tract stone management, but requires further technical improvements to match the performance of a reusable device. With their new-generation UE3022, Pusen has made significant improvements and offers a quality comparable with the LithoVue device.

\section{Authors' Contributions}

D.S.: protocol/project development, conduction of experiments, data collection and management, and data analysis. M.A.O.: data analysis and supervision and conduction of experiments. S.H.: data analysis, article and figure writing/ editing. K.W.: supervision and conduction of experiments. M.S.: data collection and management, data analysis, article and figure writing/editing. C.G.: article and figure writing/ editing and supervision. A.M.: protocol/project development, article and figure writing/editing, and supervision. D.S.S.: protocol/project development, data collection and management, data analysis, article and figure writing/editing, and supervision.

\section{Ethical Approval}

The study was approved by our local ethics committee (Project-ID: 24/17, Ethics Committee of the University Medical Center Freiburg) and was therefore performed in accordance with the ethical standards laid down in the 1964 Declaration of Helsinki and its later amendments. All participants gave their informed consent before their inclusion in the study.

\section{Author Disclosure Statement}

None of the authors has any conflict of interest or financial ties to disclose.

\section{Funding Information}

This research trial was funded, in part, by the Cook Medical Research Program.

\section{Supplementary Material}

\section{Supplementary Figure S1}

\section{References}

1. Oberlin DT, Flum AS, Bachrach L, Matulewicz RS, Flury SC. Contemporary surgical trends in the management of upper tract calculi. J Urol 2015;193:880-884.

2. Collins J, Keeley F, Timoney A. Cost analysis of flexible ureterorenoscopy. BJU Int 2004;93:1023-1026.

3. Carey RI, Martin CJ, Knego JR. Prospective evaluation of refurbished flexible ureteroscope durability seen in a large public tertiary care center with multiple surgeons. Urology 2014;84:42-45.

4. Knudsen B, Ferraro M. Digital video flexible ureteroscopy: GyrusACMI/Olympus Invisio ${ }^{\circledR}$ DUR $^{\circledR}$-D twelve month failure and repair experience. J Urol 2009;181:665.

5. Hein S, Schoenthaler M, Suarez-Ibarrola R, Mueller P-F, Schoeb D, Miernik A. Current trends in flexible ureteroscopy: An update. Aktuelle Urol 2019;50:56-62.

6. Schlager D, Hein S, Obaid MA, Wilhelm K, Miernik A, Schoenthaler M. Performance of single-use Flexorvue $v s$ reusable Boavision ureteroscope for visualization of calices and stone extraction in an artificial kidney model. J Endourol 2017;31:1139-1144.

7. Marchini GS, Batagello CA, Monga M, et al. In vitro evaluation of single-use digital flexible ureteroscopes: A practical comparison for a patient-centered approach. J Endourol 2018;32:184-191.

Address correspondence to: Daniel Schlager, MD Department of Urology Medical Center-University of Freiburg Hugstetterstr. 55 Freiburg D-79106 Germany

E-mail: daniel.schlager@uniklinik-freiburg.de

$\begin{aligned} & \text { Abbreviations Used } \\ \text { fURS } & =\text { flexible ureteroscopy } \\ \text { NASA } & =\text { National Aeronautics and Space Administration } \\ \text { TLX } & =\text { Task Load Index } \\ \text { CMOS } & =\text { complementary metal-oxide-semiconductor } \\ \text { DVI } & =\text { digital visual interface }\end{aligned}$

